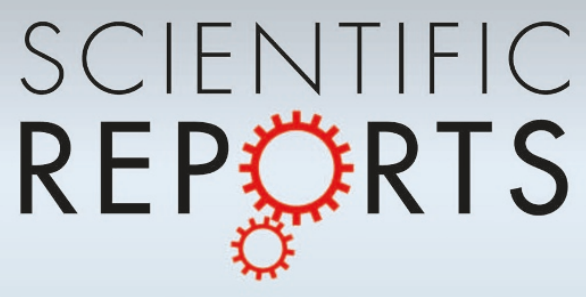

OPEN

SUBJECT AREAS:

PSYCHOLOGY

EXPERIMENTAL MODELS OF

DISEASE

Received

14 August 2013

Accepted

2 January 2014

Published

21 January 2014

Correspondence and requests for materials should be addressed to

F.X.

landrewfxu1998@ gmail.com)

\section{Perturbation of mitiglinide metabolism by chronic unpredicted mild stress in rats}

\author{
Yingtong Zeng' ${ }^{1}$ Xingqian $\mathrm{Xie}^{2}$, Jingjing Duan², Ting Zhou' ${ }^{2}$, Ye Zhang ${ }^{2}$, Min Yang ${ }^{1} \&$ Feng X ${ }^{2}$
}

'Guangdong General Hospital, Guangdong Academy of Medical Sciences, Guangzhou, 510080, China, ${ }^{2}$ Sixth People's Hospital South Campus, Shanghai Jiaotong University, Shanghai, 201400, China.

Many diabetic patients complicated with wild to severe depression. It is unclear in diabetic medication whether depression perturbs the drug metabolic process of the hypoglycemic agents or not. The present study was designed to investigate the impact of chronic unpredicted mild stress (CUMS) -induced depression on mitiglinide (MGN) pharmacokinetics in rats. Adult female Sprague-Dawley rats in CUMS group were subjected to different types of stressors and the stress procedures lasted for 8 weeks. Control group without receiving stress had free access to food and water. Open-field test and 5-HT levels were assayed to evaluate the depression. After CUMS all rats were given $2.5 \mathrm{mg} / \mathrm{kg}$ of mitiglinide per os. The blood samples were collected at different time and mitiglinide plasma concentration was measured by high performance liquid chromatography-tandem mass spectrometry (HPLC-MS/MS). Non-compartmental statistical moment analysis was processed with DAS software. In CMUS-induced depression group, peak concentration $(\mathrm{Cmax})$, peak time $(\mathrm{Tmax})$, area under curve $(\mathrm{AUC} 0 \rightarrow \infty)$, mean residence time $\left(\mathrm{MRT}_{0} \rightarrow \infty\right)$, and half-life $(\mathrm{T} 1 / 2 \mathrm{z})$ were reduced while total plasma clearance $(\mathrm{CLz} / \mathrm{F})$ was increased compared to control group. These preliminary results indicated that CUMS-induced depression alter the drug metabolic process of mitiglinide in rats. This finding will be significant in clinic.

\footnotetext{
C linically significant depression usually shares several lifestyle risk factors including smoking, physical inactivity, obesity, and excessive alcohol drinking ${ }^{1-3}$, and often coexist with medical conditions such as hypertriglyceridemia, hypertension and diabetes ${ }^{1,4-7}$. Diabetes is one of the most costly and burdensome chronic diseases, and its therapy and management have become increasingly complex. The incidence of type 2 diabetes mellitus (T2DM) is increasing at an epidemic rate worldwide. Over $10 \%$ of adults in many countries may now be affected by diabetes ${ }^{8}$. Approximately $15 \%$ of patients with diabetes mellitus meet the criteria for comorbid major depression. A bidirectional relationship between the two conditions has been recently documented in large prospective studies $^{9-14}$. People with T2DM are 15-24\% more likely to develop depression compared to people without diabetes ${ }^{15}$. It has gained much attention that the combination of diabetes mellitus and depression is associated with higher mortality rates.

Medications are needed to achieve target blood glucose levels besides healthy lifestyle choices. In most cases a combination of medications provide the foundation for managing diabetes better. Unfortunately, there are significant gaps between reaching the goal of "optimal medication therapy" and the current state of medication use $^{16}$. Despite numerous scientific and medical advances, less than half of the population with T2DM has achieved the American Diabetes Association-recommended glycated hemoglobin level goal of $<7 \%$, which is necessary to optimally manage the disease to prevent and minimize complications. There are many patient- and cliniciandetermined barriers that hinder patients from achieving target blood glucose levels ${ }^{17}$. Diabetes medication use with caution is of importance for "optimal medication therapy".

Mitiglinide (MGN), (-)-2(S)-benzyl-4-(cis-perhydroisoindol-2-yl) butyric acid, is an effective insulinotropic agent of the glinides with rapid onset. MGN is thought to stimulate insulin secretion by closing the ATP-sensitive $\mathrm{K}^{+}\left(\mathrm{K}_{\mathrm{ATP}}\right)$ channels in pancreatic beta cells ${ }^{18}$. Its early insulin release and short duration of action is effective to improve postprandial hyperglycemia ${ }^{19}$. Currently MGN is an ideal drug to treat type 2 diabetes and is widely used in clinical practice.

Drug efficacy is chiefly determined by its physicochemical properties and pharmacological effects. Besides, many non-pharmacological factors such as age, gender, psychological/social elements also impact the overall therapeutic outcome ${ }^{20}$. It is a common phenomenon that depressive disorder perturbs the drug efficacy in clinical practice $^{21,22}$. However, it is unclear whether depression perturbs the drug metabolic process of the hypoglycemic agents in diabetic medication or not. The present study was designed to investigate the impact of chronic unpredicted mild stress (CUMS) -induced depression on MGN pharmacokinetics in rats.
} 


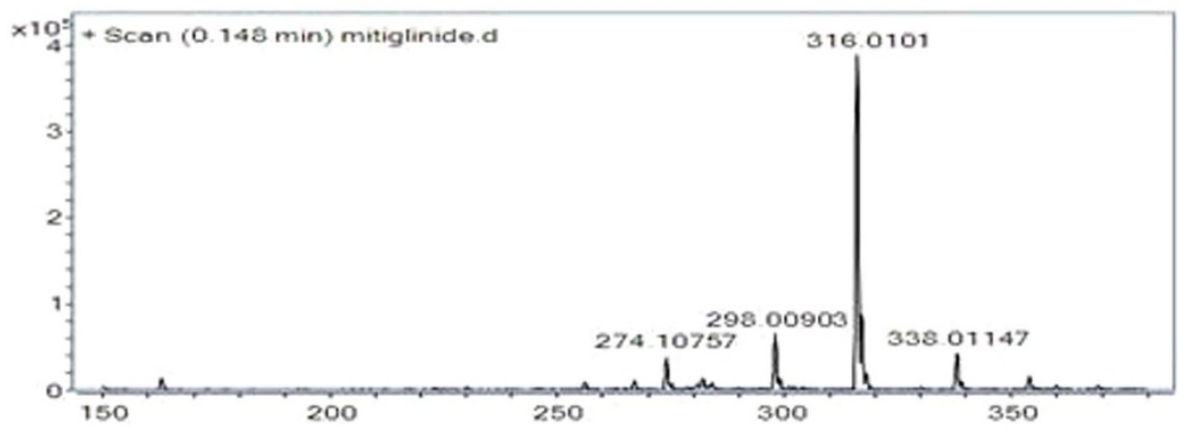

Figure 1 | Full-scan positive ESI-MS/MS production spectra mitiglinide (316 ->298).

\section{Results}

Validation of CUMS-induced depression ${ }^{23,24}$. The locomotion and exploratory behavior scores of rats in CUMS-induced depression group and control group before and after 8 weeks' model establishment were monitored through open-field test ${ }^{25}$. The baseline locomotion and exploratory scores between the groups were same but significantly different at the end of 8 weeks $(\mathrm{p}<$ 0.01). Within CUMS-induced depression group, the locomotion and exploratory scores of rats were decreased from $78.67 \pm 6.91$ to $15.22 \pm 4.71(\mathrm{p}<0.01)$, and from $15.89 \pm 2.80$ to $4.89 \pm 1.69(\mathrm{p}<$ 0.01 ), respectively. No significant change for the locomotion and exploratory scores occurred within control group, which were from $77.22 \pm 6.10$ to $70.89 \pm 8.91(\mathrm{p}>0.05)$, from $14.67 \pm 2.87$ to $15.22 \pm 5.52(\mathrm{p}>0.05)$, respectively.

5-HT plasma levels ${ }^{26}$ of rats in CUMS-induced depression group before and after 8 weeks' model establishment were significantly decreased from $6.74 \pm 2.64$ to $2.22 \pm 0.75 \mathrm{ng} / \mathrm{ml}(\mathrm{p}<0.01)$, and no change in control group (from $5.60 \pm 1.76$ to $5.59 \pm 2.09 \mathrm{ng} / \mathrm{ml}$ ) $(\mathrm{p}>0.05)$. There was significant difference for 5 -HT levels occurred between the two groups at the 8 weeks end $(2.22 \pm 0.75$ vs $5.59 \pm$ $2.09 \mathrm{ng} / \mathrm{ml})(\mathrm{p}<0.01)$.

Pharmacokinetic moment analysis of MGN. MGN plasma concentration was determined by HPLC-MS/MS. The assay validation for MGN is summarized in Figure 1, Figure 2 and Table 1. The plasma concentration- time data of MGN were calculated for statistical moment analysis with DAS 2.1 software (Drug and Statistics, China) (Table 2). Peak concentration (Cmax) and peak time (Tmax) were reduced a little in CUMS-induced depression group compared to those in control group, while area under curve $(\mathrm{AUC} 0 \rightarrow \infty)$, mean residence time $(\mathrm{MRT} 0 \rightarrow \infty)$, and half-life $(\mathrm{T} 1 / 2 \mathrm{z})$ decreased significantly $(\mathrm{P}<0.05)$. Total plasma clearance $(\mathrm{CLz} / \mathrm{F})$ was extended significantly compared to that in control group $(\mathrm{P}<0.05)$.

\section{Discussion}

An ideal animal model should be able to simulate the development process of diseases and the change of pathophysiology. Chronic
A
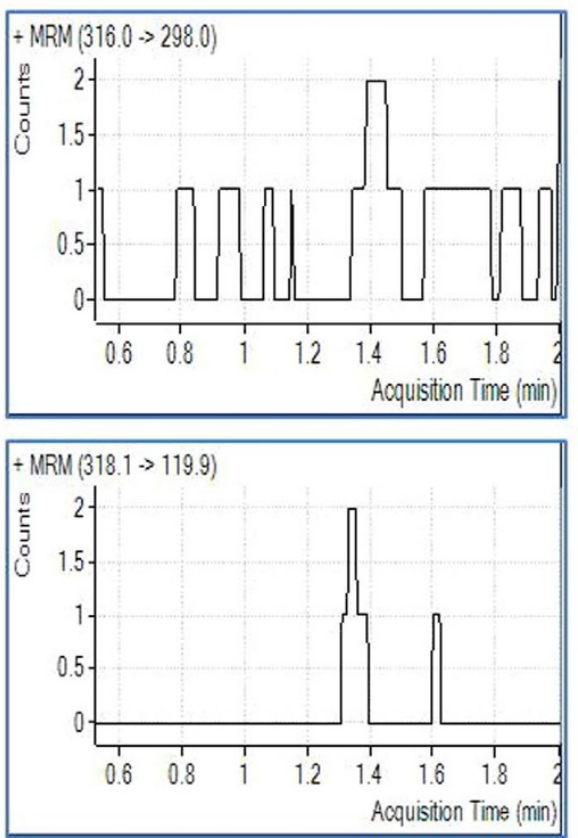

A: blank plasma
B
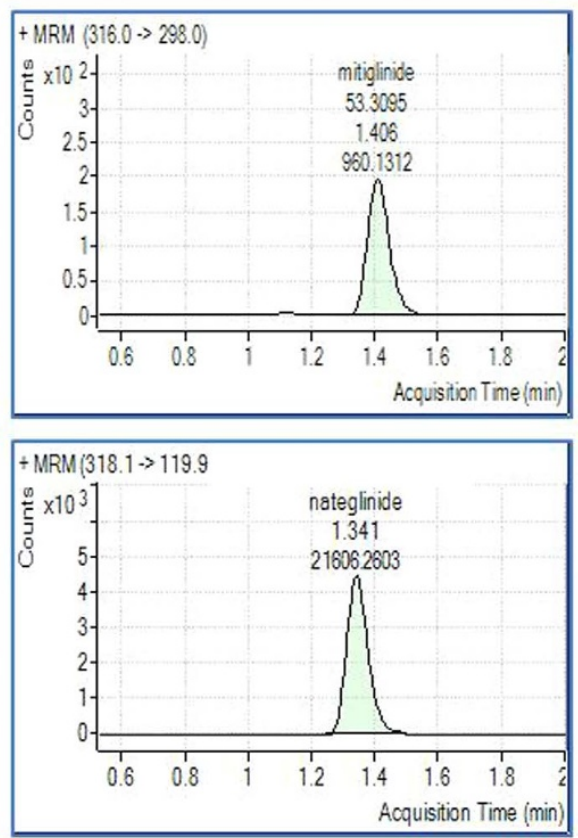

B: plasma spiked with MGN/IS
C
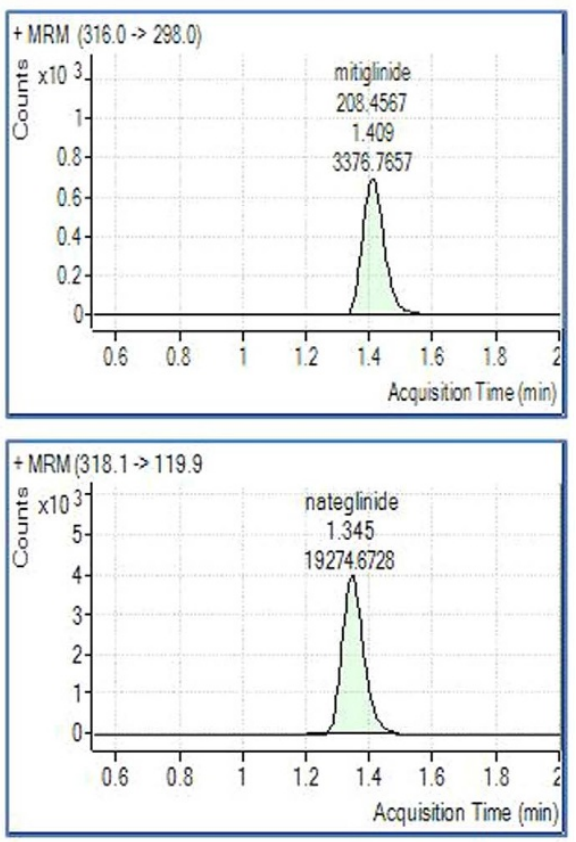

C: rat plasma sample

Figure $2 \mid$ Typical chromatograms of mitiglinide (Top, retention time $1.40 \mathrm{~min}$.) and internal standard nateglinide (Bottom, retention time $1.30 \mathrm{~min}$.) in blank plasma, plasma spiked with mitiglinide/nateglinide, and rat plasma sample. 
Table 1 | Recovery, matrix effect, precision and accuracy for the determination of MGN in plasma by HPLC-MS/MS

\begin{tabular}{lcccccc}
$\begin{array}{l}\text { Nominal concentration } \\
(\mu \mathrm{g} / \mathrm{ml})\end{array}$ & Recovery (\%) & $\begin{array}{c}\text { Measured concentration } \\
(\mu \mathrm{g} / \mathrm{ml})\end{array}$ & Accuracy (\%) & Matrix effect (\%) & $\begin{array}{c}\text { Intra-day precision } \\
\text { (RSD\%) }\end{array}$ & $\begin{array}{c}\text { Inter-day precision } \\
\text { (RSD\%) }\end{array}$ \\
\hline 2.5 & $92.27 \pm 0.66$ & $2.603 \pm 0.104$ & 104.13 & $93.76 \pm 5.57$ & 4.32 & 7.53 \\
1 & $91.60 \pm 4.90$ & $1.039 \pm 0.040$ & 103.90 & $96.57 \pm 1.14$ & 4.19 & 6.67 \\
0.05 & $98.64 \pm 6.66$ & $0.049 \pm 0.002$ & 98.75 & $93.89 \pm 9.06$ & 3.82 & 5.18 \\
\hline
\end{tabular}

Notes: Data are based on analysis of six replicates $(n=6)$ on three separate days.

unpredictable mild stress (CUMS), a well-validated animal model, has been used widely for depression research as well as antidepressant evaluation for many years ${ }^{27}$. In CUMS-induced depression rats, the depressive state is similar with change of psychomotor and loss of interest or pleasure in the clinical diagnosis of depression ${ }^{28-30}$. Our results verified the significant $5-\mathrm{HT}$ and psychomotor decrease after depression model establishment in CUMS-induced depression group. In this study, female rats were adopted in this animal model because female rats are more vulnerable in an animal model of depression compared to male rats ${ }^{31}$. Although in CUMS-induced depression group the stressors were mentioned to be used randomly, the CUMS protocol should be the same every time. Since each stressor has its own short and long term physiological and biochemical consequences, in order to maintain reproducibility and consistency of finding similar stress regime should be followed.

MGN plasma level was determined using the HPLC-MS-MS method in this study. There was no significant interference or ion suppression from endogenous substances observed at the retention time of the analytes. The retention times for MGN and internal standard nateglinide were 1.4 and $1.3 \mathrm{~min}$., respectively. An sevenpoint calibration curve obtained by weighted linear regression $\left(1 / \chi^{2}\right)$ showed good linearity over the concentration range of plasma ( 0.05 $\sim 3.5 \mu \mathrm{g} / \mathrm{ml}$ ), which covered the concentrations typically found in plasma after administration of MGN in the pharmacokinetic study. The lower limit of quantification was $0.05 \mu \mathrm{g} / \mathrm{ml}$. The HPLC-MSMS method validation showed satisfactory determination of MGN in plasma and could be used for pharmacokinetic studies in rats.

Significant differences in some pharmacokinetic moment parameters of MGN between two groups were described in this study for first time. MGN metabolic process in CUMS-induced depression group was speeded up compared to control group. The perturbation was speculated to be mediated by altering drug-metabolizing enzymes expression and/or activity. Our pilot experimental animal study found that CUMS-induced depression increased CYP450 total levels and enhanced the enzyme activity in rat liver tissues ${ }^{32}$. Other researchers revealed that the genotype and phenotype of CYP2D6 had individual differences which were related to personality traits. The hydroxylation capacity of CYP2D6 is associated with the level of anxiety and the degree of socialization of patients ${ }^{33,34}$. These dada suggested that depressive disorder could alter the expression and activity of some drug-metabolizing enzymes which metabolized MGN in human body. UGT1A3, UGT1A9 and UGT2B7 were important catalytic enzymes in MGN carboxyl-glucuronidation in

Table 2 | The main pharmacokinetic moment parameters of MGN

\begin{tabular}{llcc} 
Parameter & \multicolumn{1}{c}{$\begin{array}{c}\text { Control group } \\
(\mathrm{n}=9)\end{array}$} & $\begin{array}{c}\text { Depression group } \\
(\mathrm{n}=9)\end{array}$ \\
\hline $\mathrm{Cmax}$ & $\mathrm{ug} / \mathrm{mL}$ & $1.88 \pm 0.23$ & $1.84 \pm 0.93$ \\
$\mathrm{Tmax}$ & $\mathrm{min}$ & $23.00 \pm 25.30$ & $22.22 \pm 14.17$ \\
$\mathrm{AUC}(0-\infty)$ & $\mathrm{ug} / \mathrm{mL}{ }^{*} \mathrm{~min}$ & $501.79 \pm 68.24$ & $475.16 \pm 62.01^{*}$ \\
$\mathrm{MRT}(0-\infty)$ & $\mathrm{min}$ & $367.02 \pm 85.84$ & $245.95 \pm 68.83^{*}$ \\
$\mathrm{tl} / 2 z$ & $\mathrm{~min}$ & $256.12 \pm 48.50$ & $168.10 \pm 34.68 *$ \\
$\mathrm{CLz} / \mathrm{F}$ & $\mathrm{mL} / \mathrm{min}$ & $0.83 \pm 0.08$ & $0.96 \pm 0.11^{*}$ \\
\hline Notes: ${ }^{*} \mathrm{p}<0.05$ compared with control group. &
\end{tabular}

human liver ${ }^{35,36}$. This study did not analyze these three specific enzymes due to many limitations. Besides, it will be better if the pharmacokinetics of MGN was studied in human body. Nevertheless, our preliminary results warn that potential MGN pharmacokinetic alteration might occur in diabetic patients complicated with depression. It might be necessary to adjust the MGN dose in long-term diabetes medication treatment. Whether and how CUMSinduced depression alters UGT1A3, UGT1A9 and UGT2B7 level and/or activity in rats is worth studying in future.

\section{Methods}

Animals. Adult female Sprague-Dawley (SD) rats (Laboratory Animal Center of Southern Medical University, Animal license: SCXK 2006-0015) weighing 150-200 g were kept in an animal colony at a density of approximately 5-6 per cage for 2 weeks prior to the experiment. All experiments were conducted between 9:00 a.m. to 12:00 p.m. under standard laboratory conditions $\left(23 \pm 2{ }^{\circ} \mathrm{C}\right.$ room temperature; $12 \mathrm{hr}$ light/ dark cycle with lights on at 7:00 a.m.). Tap water and food pellets were provided ad libitum. All animals used in this study were naive to the experimental test. Animals were divided into two groups ( $\mathrm{n}=9$ per group): control group and CUMS group.

Chronic unpredictable mild stress procedure. Chronic unpredictable mild stress was applied as previously described by Willner et al. ${ }^{24}$ with a minor modification. Briefly, animals in CUMS group were single cage bred and subjected to different types of stressors: restraint for $1 \mathrm{hr}$, cage tilting for $24 \mathrm{hr}$, damp sawdust bedding for $24 \mathrm{hr}$, swimming in $4^{\circ} \mathrm{C}$ cold water for $5 \mathrm{~min}$., swimming in $45^{\circ} \mathrm{C}$ hot water for $5 \mathrm{~min}$., nip tail for $1 \mathrm{~min}$., level shaking for $10 \mathrm{~min}$., noise stimulus at $11 \mathrm{db}$ for $10 \mathrm{~min}$., pairing with another stressed animal for $24 \mathrm{hr}$, and inversion of the light/dark cycle for $24 \mathrm{hr}$. These ten stressors were randomly applied for 8 weeks, and each stressor was applied 6-7 times during this time period. Rats received one of these stressors per day. In order to avoid habituation the same stressor was not applied consecutively for 2 days so that the animals could not predict the occurrence of stimulation. CUMS protocol was the same every time to maintain reproducibility and consistency of finding in future. The stress procedure did not involve any food or water deprivation in this study. During the stress process, rat was moved into special room to accept the stressor and returned its single cage after accepting the stressor. The control group receiving no stress had free access to food and water.

Open-field test. The apparatus for the open-field test was a box $(76 \times 76 \times 42 \mathrm{~cm})$ made of opaque materials. The open-field arena was partitioned into 25 equal-size squares. The test was conducted in a quiet room in the morning $(8: 00 \sim 12: 00$ a.m. $)$ Each rat was placed in the center of the arena and its behavior was recorded for $5 \mathrm{~min}$. Four claws climbing square numbers and rearing times were monitored as an index of locomotor activity and exploratory behavior. The open-field was cleaned after each test.

Determination of 5-HT plasma level. About $0.5 \mathrm{ml}$ of blood was collected from rat at entry and at the end of 8 weeks of model establishment. The anticoagulated blood sample was centrifuged at $3000 \times \mathrm{g}$ for $5 \mathrm{~min}$. to obtain plasma. The level of $5-\mathrm{HT}$ in plasma was tested by enzyme-linked immunosorbent assay (ELISA).

Dosage regimen and determination of MGN concentration in plasma. After depression model establishment, rats in both groups were given $2.5 \mathrm{mg} / \mathrm{kg}$ MGN per os. Blood was collected at different time and MGN concentration was assayed by HPLC-MS/MS. Briefly, a volume of $2 \mathrm{ml}$ ethyl acetate and $0.1 \mathrm{ml}(5 \%)$ methanoic acid were added to a centrifuge tube, in which $200 \mu \mathrm{l}$ plasma sample and $50 \mu \mathrm{l}$ of the internal standard (nateglinide, $2 \mu \mathrm{g} / \mathrm{ml}$ ) were placed. The sample was vortex-mixed vigorously for $1 \mathrm{~min}$., followed by centrifugation at $3000 \times \mathrm{g}$ for $10 \mathrm{~min}$. The organic layer was collected and evaporated to dryness at $42^{\circ} \mathrm{C}$ under a gentle stream of nitrogen. The residue was re-dissolved in $200 \mu \mathrm{l}$ mobile phase and vortex-mixed vigorously for $1 \mathrm{~min}$. again, followed by centrifugation at $3500 \times \mathrm{g}$ for $10 \mathrm{~min}$. A volume of $1 \mu \mathrm{l}$ of the supernatant was directly injected into the HPLC-MS/MS system. HPLC-MS/MS instrumentation and conditions for determination of MGN were: the LC system was consisted of an Agilent 1200 series G1310A pump, a G1310A degasser, a G1329A auto-sampler and a G1316A adjustable column temperature box. The chromatography of the analyte was performed at $40^{\circ} \mathrm{C}$ using an Agilent ZORBAX SB-C18 $(2.1 \mathrm{~mm} \times 150 \mathrm{~mm}, 5 \mu \mathrm{m})$ column. The flow rate of mobile phase 
(methanol: $0.1 \mathrm{~mol} / \mathrm{l}$ ammonium formate, $95: 5(\mathrm{v} / \mathrm{v})$ ) was $0.5 \mathrm{ml} / \mathrm{min}$. The optimized conditions of MS/MS with electrospray were as follows: ion spray source temperature at $350^{\circ} \mathrm{C}$, nebulizer (NEB) gas at $10 \mathrm{~L} / \mathrm{min}$, ionspray voltage (IS) at $4000 \mathrm{~V}$. Dissociating potential (DP) for MGN was at $145 \mathrm{~V}$ and collision energy (CE) was 11 units, dissociating potential for nateglinide was at $95 \mathrm{~V}$ and collision energy (CE) was 20 units. The mass spectrometer was interfaced to a computer workstation running Aria ${ }^{\circledR}$ OS software and Analyst software (Version B.01.03 Applied Biosystems) for data acquisition and processing. Data acquisition was performed via multiple-reaction monitoring (MRM). The ions representing the $[\mathrm{M}+\mathrm{H}]^{+}$species for both the sample and internal standard were selected in MS1 and dissociated (collision-induced) with nitrogen gas to form specific product ions, which were subsequently monitored by MS2. The precursor-to-product ion transitions monitored for MGN and nateglinide were $\mathrm{m} / \mathrm{z} 316.0 \rightarrow 298.0$ and $\mathrm{m} / \mathrm{z} 318.1 \rightarrow 119.9$ respectively.

Statistical analysis. Statistical analysis was performed with the SPSS 16.0 (SPSS Inc. Chicago, IL, USA) and DAS 2.1 software (Drug and Statistics, Shanghai University of Traditional Chinese Medicine, Shanghai, China). Data were expressed as mean \pm SD. $\mathrm{p}<0.05$ was considered statistically significant.

Ethics statement. The animal experiment protocol was approved by the Bioethics Committee of Guangdong General Hospital, the institution hosting the experiment.

1. Atlantis, E. et al. Specific medical conditions associated with clinically significant depressive symptoms in men. Soc. Psychiatry. Psychiatr. Epidemiol. 46, 1303-1312 (2011)

2. Strine, T. W. et al. The association of depression and anxiety with obesity and unhealthy behaviors among community-dwelling US adults. Gen. Hosp. Psychiatry 30, 127-137 (2008).

3. Bonnet, F. et al. Anxiety and depression are associated with unhealthy lifestyle in patients at risk of cardiovascular disease. Atherosclerosis 178, 339-344 (2005).

4. Grimsrud, A., Stein, D. J., Seedat, S., Williams, D. \& Myer, L. The association between hypertension and depression and anxiety disorders: results from a nationally-representative sample of South African adults. PLoS. One 4, e5552 (2009).

5. Pietraszek, A., Gregersen, S. \& Hermansen, K. Alcohol and type 2 diabetes. A review. Nutr. Metab. Cardiovasc. Dis. 20, 366-375 (2010).

6. Atlantis, E., Lange, K. \& Wittert, G. A. Chronic disease trends due to excess body weight in Australia. Obes. Rev. 10, 543-553 (2009).

7. Magliano, D. J. et al. Glucose indices, health behaviors, and incidence of diabetes in Australia: the Australian Diabetes, Obesity and Lifestyle Study. Diabetes. Care 31, 267-272 (2008).

8. Atlantis, E., Vogelzangs, N., Cashman, K. \& Penninx, B. J. Common mental disorders associated with 2-year diabetes incidence: the Netherlands Study of Depression and Anxiety (NESDA). J. Affect. Disord. 142, S30-S35 (2012).

9. Vijan, S. Type 2 diabetes. Ann. Intern. Med. 152, C15-C31, C316 (2010).

10. Golden, S. H. et al. Examining a bidirectional association between depressive symptoms and diabetes. JAMA. 299, 2751-2759 (2008).

11. Katon, W., Lin, E. H. \& Kroenke, K. The association of depression and anxiety with medical symptom burden in patients with chronic medical illness. Gen. Hosp. Psychiatry 29, 147-155 (2007).

12. Katon, W. J. The comorbidity of diabetes mellitus and depression. Am. J. Med. 121, S8-S15 (2008).

13. Ciechanowski, P. S., Katon, W. J. \& Russo, J. E. Depression and diabetes: impact of depressive symptoms on adherence, function, and costs. Arch. Intern. Med. 160, 3278-3285 (2000)

14. Murray, C. J. \& Lopez, A. D. Global mortality, disability, and the contribution of risk factors: Global Burden of Disease Study. Lancet 349, 1436-1442 (1997).

15. van Dooren, F. E. et al. Depression and risk of mortality in people with diabetes mellitus: a systematic review and meta-analysis. PLoS. One 8, e57058 (2013).

16. Smith, M. Pharmacist's role in improving diabetes medication management. J. Diabetes Sci. Technol. 3, 175-179 (2009).

17. Cornell, S. \& Dorsey, V. J. Diabetes pharmacotherapy in 2012: considerations in medication selection. Postgrad. Med. 124, 84-94 (2012).

18. Sunaga, Y. et al. The effects of mitiglinide (KAD-1229), a new anti-diabetic drug, on ATP-sensitive $\mathrm{K}+$ channels and insulin secretion: comparison with the sulfonylureas and nateglinide. Eur. J. Pharmacol. 431, 119-125 (2001).
19. Ohnota, H. et al. A rapid- and short-acting hypoglycemic agent KAD-1229 improves post-prandial hyperglycemia and diabetic complications in streptozotocin-induced non-insulin-dependent diabetes mellitus rats. Jpn. J. Pharmacol. 71, 315-323 (1996).

20. Xu, J., Mercury, J., Zhang, Z. \& Xu, F. Psychological, social and behavioral factors that influence drug efficacy: a noteworthy research subject in clinical pharmacology. Br. J. Clin. Pharmacol. 66, 901-902 (2008).

21. Xu, F. Effect of personality type on pharmacodynamics through changing pharmacokinetics. Med. Hypotheses. 69, 1131-1134 (2007).

22. Peng, H. T. \& Cheung, B. A review of psychophysiological stressors on pharmacokinetics. J. Clin. Pharmacol. 51, 1499-1518 (2011).

23. Katz, R. J., Roth, K. A. \& Carroll, B. J. Acute and chronic stress effects on open field activity in the rat: implications for a model of depression. Neurosci. Biobehav. Rev. 5, 247-251 (1981).

24. Willner, P., Towell, A., Sampson, D., Sophokleous, S. \& Muscat, R. Reduction of sucrose preference by chronic unpredictable mild stress, and its restoration by a tricyclic antidepressant. Psychopharmacology (Berl). 93, 358-364 (1987).

25. Kennett, G. A., Dickinson, S. L. \& Curzon, G. Enhancement of some 5-HTdependent behavioral responses following repeated immobilization in rats. Brain. Res. 330, 253-263 (1985).

26. Schafer, A. et al. Platelet serotonin (5-HT) levels in interferon-treated patients with hepatitis $\mathrm{C}$ and its possible association with interferon-induced depression. J. Hepatol. 52, 10-15 (2010).

27. Papp, M., Willner, P. \& Muscat, R. An animal model of anhedonia: attenuation of sucrose consumption and place preference conditioning by chronic unpredictable mild stress. Psychopharmacology (Berl). 104, 255-259 (1991).

28. Willner, P. Validity, reliability and utility of the chronic mild stress model of depression: a 10-year review and evaluation. Psychopharmacology (Berl). 134 319-329 (1997).

29. Liu, M., Hu, J., Ma, T., Wang, S. \& Ding, H. Application of a disposable screenprinted electrode to depression diagnosis for laboratory rats based on blood serotonin detection. Anal. Sci. 27, 839-843 (2011).

30. Spasojevic, N., Gavrilovic, L., Kovacevic, I. \& Dronjak, S. Effects of antidepressants maprotiline and fluxilan on sympatho-adrenomedullary system in stressed rats. Auton. Neurosci. 145, 104-107 (2009).

31. Kennett, G. A., Chaouloff, F., Marcou, M. \& Curzon, G. Female rats are more vulnerable than males in an animal model of depression: the possible role of serotonin. Brain. Res. 382, 416-421 (1986).

32. Duan, J. J. et al. Pharmacokinetics of 5-fluorouracil and cyclophosphamide in depression rats. Eur. Rev. Med. Pharmacol. Sci. 16, 427-436 (2012).

33. Dorado, P., Penas-Lledo, E. M. \& Llerena, A. CYP2D6 polymorphism: implications for antipsychotic drug response, schizophrenia and personality traits. Pharmacogenomics 8, 1597-1608 (2007)

34. Gonzalez, I. et al. Relation between CYP2D6 phenotype and genotype and personality in healthy volunteers. Pharmacogenomics 9, 833-840 (2008).

35. Jin, L. \& Cao, Q. Effect of gemfibrozil on the pharmacokinetics of mitiglinide in rats. Arzneimittelforschung 62, 40-45 (2012).

36. Yu, L., Lu, S., Lin, Y. \& Zeng, S. Carboxyl-glucuronidation of mitiglinide by human UDP-glucuronosyltransferases. Biochem. Pharmacol. 73, 1842-1851 (2007).

\section{Author contributions}

Y.T.Z., X.Q.X., J.J.D. and T.Z. conducted the animal experiment. Y.T.Z., X.Q.X. and J.J.D. wrote the main manuscript. T.Z. analyzed the data. Y.Z. and M.Y. prepared the figures. F.X. conceived and designed the experiment and revised the manuscript. All author reviewed the manuscript.

\section{Additional information}

Competing financial interests: The authors declare no competing financial interests.

How to cite this article: Zeng, Y.T. et al. Perturbation of mitiglinide metabolism by chronic unpredicted mild stress in rats. Sci. Rep. 4, 3794; DOI:10.1038/srep03794 (2014).

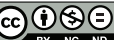

This work is licensed under a Creative Commons AttributionNonCommercial-NoDerivs 3.0 Unported license. To view a copy of this license, visit http://creativecommons.org/licenses/by-nc-nd/3.0 